\title{
Mechanical Part Surface Defect Detection using Crack Extraction Approach
}

\author{
Priyanka Khandelwal \\ PG Student, \\ Computer Science \& Engg. Dept. \\ Gujarat Technological University, India
}

\author{
Pankaj Kumar Gautam \\ Assistant Professor, \\ Computer Science \& Engg. Dept. \\ Parul Group of Institute, India
}

\begin{abstract}
Visual inspection constitutes an important part of quality control in manufacturing industry. The detection of defects on mechanical part surfaces is an important quality control step in the manufacturing of machine products. In this paper, we have introduced a new approach to detect surface defects with varied size, shape in mechanical parts through the use of image processing techniques. First, we apply image edge detection techniques for extracting the edges in an image by identifying pixels where intensity variation is high. Then, for extracting actual defects we reduce gray scale edge information to binary defect information using thresholding. A threshold process will generate a certain amount of noise. So, this noise will removed by a noise filtering technique using the connected component's eccentricity property. Then, based on the highlighted edges, the defect itself should become identifiable by filling the gap between two corresponding edges by comparing gray scale values. The Experimental results show that the proposed method is suitable for extracting the various defects of varying shapes and size in images.
\end{abstract}

\section{Keywords}

Defect Detection, Crack Extraction, Edge Detection, Thresholding, Connected Component Property, Gray Scale values

\section{INTRODUCTION}

Digital image processing is used to extract various features from images. This is done by computers automatically without or with little human intervention. One of the most important operations on digital image is to recognize and classify various kinds of defects. Thus to detect the defects from any image various techniques are established and placed at three levels. At the lowest level, some algorithms are available which deal directly with the raw, possibly noisy pixel values, with de-noising and edge detection being good examples. In the middle, there are techniques which utilize low level results, such as segmentation and edge linking. At the highest level are those techniques which attempt to extract semantic meaning from the information provided by the lower level [1].

Quality control is an important part in machine product manufacturing process. Therefore, production inspection should be carried out to evaluate the quality of products [2]. The quality assurance of product is mainly carried out by manual inspection. However, the reliability of manual inspection is limited by ensuing fatigue and inattentiveness. It is proved that only about $70 \%$ of the defects could be detected by the most highly trained inspectors [4]. With the exponential growth of technological era the manual inspection systems has been drastically brought down. Decades ago this manual inspection systems have been replaced by automated visual inspection systems. There are many visual inspection systems which are used for defect detection of textured material [3], textile fabric [4], and fruits [6] etc. In the past years, some of the defect detection techniques have been proposed and they are classified in four different approaches. These approaches are statistical, model based, structural and filter based. Various defect detection techniques based on these approaches for defect detection in various types of images are as follows: independent component analysis (ICA) for textile fabric images [5] , Optimal Gabor filtering [6], feature extraction and segmentation method for texture images [7], Gabor wavelet filter and Gaussian filter to detect the defects in digital image [9] and Otsu method based on morphology and wavelet transform to detect surface defects of parts [10].But in existing techniques, they are limited to predefined defect type and doesn't give the good result in detecting defects of varying shapes and sizes in images.

The objective of research is to propose an approach (i.e. Crack Extraction) which will be able to extract the thin defect (i.e. Crack) as well as multiple defects of varying shapes and sizes at high detection rate in mechanical part images. The proposed algorithm is developed through the use of image processing techniques like edge detection, thresholding, noise filtering techniques, and connected component property.

This paper is organized as follows: Section 2 reviews the various existing techniques of defect detection briefly. Section 3 illustrates proposed Technique. i.e. Crack Extraction. Section 4 shows the experimental results. Finally, the conclusion is presented in Section 5.

\section{EXISTING DEFECT DETECTION TECHNIQUES}

In the previous years, some defect detection techniques have been proposed to find out the various image defects. But they have some limitations that can be described briefly as follows:

Hamid Alimohamdi et al. [6] presented method based on optimal Gabor filter for online defects detection in various fruits. This technique is robust and computationally efficient for detection of fruits defects. This algorithm is also considered the selection of filter parameters, especially center frequency and mask size, which heavily relates to the texture characteristics. The estimate of parameters is important for improving detection rates and reducing false alarm rates. The parameter used in this algorithm is not working very well on every individual image. If this algorithm uses histogram equalization techniques than it can't detect the defect which have limited depth. 
K. N. Sivabalan et al. [7] Adopted the technique of feature extraction and segmentation to identify the defects in the digital image. This proposed defect detection technique which is fast and simple compared to other defect detection algorithms. This Algorithm has the capacity to be used in various types of images. This Algorithm is most suitable for the defects which have low frequency. The drawback of this algorithm is that the method is not suitable for all forms of defects and the efficiency of this algorithm is low [8]. Each and every defect pixel are not identified by this algorithm.

K. N. Sivabalan et al. [9] Presented defect detection technique using Gabor wavelet filter and Gaussian filter to identify the defects in various digital images in industries. This Algorithm is used to identify the defects in the digital texture image using non texture methods. The Algorithm has proved to be $85 \%$ efficient in detecting the defects. The drawback of this algorithm is that the method cannot identify defects in high intensity levels and shape of the defect is not properly detected.

Gui-mei et al. [10] Presented a new segmentation algorithm to detect the surface defect of parts, which applied with morphology and wavelet transform into the Otsu algorithm. The method can effectively extract the defect from the images which contain complex background and noise. This algorithm has higher segmentation accuracy and better noise resistance than that of the traditional one and two dimensional Otsu. More than one defect from an image is not effectively extracted by this algorithm.

So, from existing techniques of defect detection, there is still the problem of detecting the various defects of varying shape in images and also doesn't give the best result of detecting the true shape of the defects of different size, orientation.

\section{PROPOSED APPROACH FOR DEFECT DETECTION}

In this section, we describe the proposed approach which is able to extract the crack (i.e. Thin defect) as well as defects with varied shape in images of mechanical parts. The steps of the proposed algorithm are shown in the flow chart (see Figure 1) and also describe the each and every step of the proposed approach (i.e. Crack extraction) in the following sections.

\subsection{Edge Detection}

After the Preprocessing step, we apply the edge detection method to an image which may specially reduce the amount of data to be processed and filter out information that are less relevant, while at the same time preserving useful information about the boundaries. Thus, edge detection provides basic information which is used for extracting shapes. It is used to determine the each \& every edge in an image. There are many edge detector operators like Sobel, Prewitt, Roberts, LOG (Laplacian of Gaussian) and Canny Operator [11]. Compared to other methods, Canny is one of the most commonly used optimal edge detector to find edges by isolating noise from the image. It keeps a good balance between noise and edge detection and not easily disturbed by noise. It has advantages to give good detection, good localization and minimal response [12].

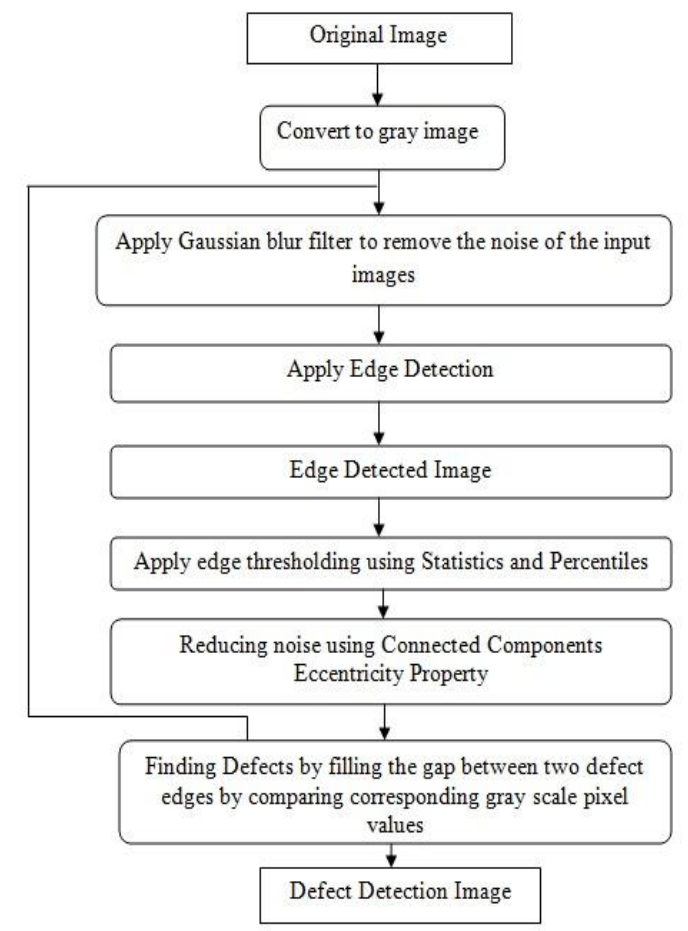

Fig 1: Flow of the proposed work

\subsection{Edge Thresholding using Statistics and Percentiles}

After the above step, we have an edge detected image. In order to identify defects pixel and non-defect pixel in edge image, we performed the edge thresholding on edge detected image. During the thresholding process, individual pixels in the edge image are marked as defect pixels if their value is larger than some threshold value and as background (nondefect) pixels otherwise. Finally, a binary defect image is created by coloring each pixel either black or white, depending on whether it is marked as a defect pixel or a background pixel. Therefore, and in order to get a threshold value that best represents the underlying edge image, statistics and percentiles [13] are applied. In statistics, a percentile provides an indication of how the data values, sorted from smallest to largest, are spread.

\subsection{Noise Filtering using Connected Components Eccentricity Property}

A thresholding process will generate a certain amount of noise. This noise pixel has abnormal intensity pixels. In detail, a black defect pixel may appear in the middle of a white nondefect area. This is treated as noise and it's not desirable. Therefore, a method for removing this noise is implemented in following ways. First of all connected components in the binary image is found using 8- connectivity neighborhood. This means that binary image is processed as pixel by pixel, grouping all adjacent defect pixels into one component. Each connected component is then measured according to its eccentricity property. The eccentricity value is between 0 and 1. The ellipse whose eccentricity is ' 0 ' is a circle while ' 1 ' is a line segment. By taking advantage of this fact and by simply comparing a connected component's eccentricity value against a specified value between 0 and 1 , it becomes possible to remove complete circle-like components. This is focused to make in a line segment instead of a circle like components. 


\subsection{Finding defects by filling the gap between two defect edges by comparing corresponding gray scale pixel values}

The last step of the crack extraction process consists of simple comparisons between gray scale values. Every defect pixel has a corresponding gray scale value found in the underlying gray scale image. Here, all defect pixels are in fact binary edge pixel. Therefore, for finding defects, filling the appropriate gap between two corresponding binary edge pixels is a twofold iterative approach where the first iteration differs from the rest.

In the first iteration, moving one pixel away from the edge and thus into the defect. During the first iteration, a defect (binary edge) pixel compares its neighbors gray scale values against each other in a total of four ways. The grayscale value of its left neighbor is compared to the grayscale value of its right neighbor. The neighbor that has the smallest value is marked as a new defect pixel if and only if its value is also smaller than the current pixel gray scale value. The same logic applies to the other opposite neighbors. As a result, all binary edges are expanded by one pixel into the defect. The remaining iterations are different. It's like in edge thresholding; based on the values in the grayscale image, percentiles are here used to find a small value. In order to be marked as a defect pixel, the grayscale value of a pixel needs to be lower than this percentile value. That way, it becomes feasible to simply pick every neighbor with a gray scale value smaller than the pre-defined defect value. Considering that every defect pixel is now in fact located inside an actual defect. Finally, after all these iterations, we have a defect extracted output image.

\section{EXPERIMENTAL RESULTS}

To prove the effectiveness of the proposed approach, we conduct experiments on various mechanical part images with varied size, which contain different defects like spot, crack, blow hole etc. of varying shape. The Test image 1 which of size $200 \times 149$ consists of a defect in mechanical part. Figure 2 shows the test result1, (a) original image (b) preprocessed

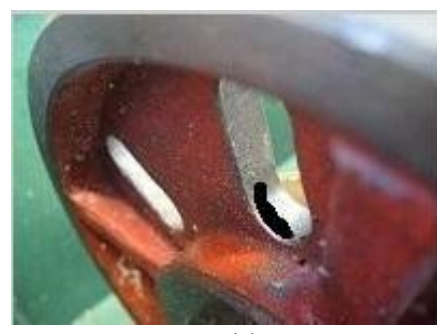

(a)

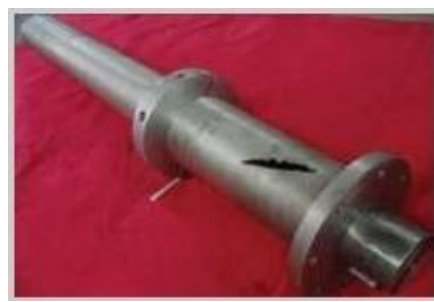

(e)

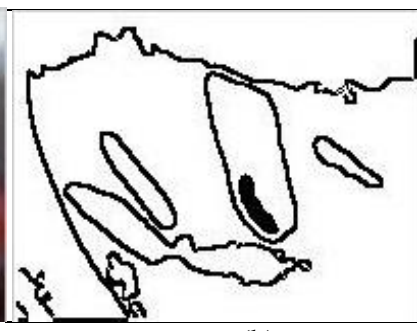

(b)

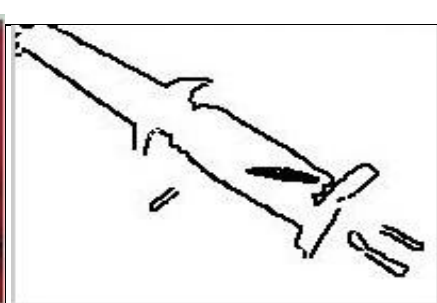

(f)

Fig 3: Experimental results of proposed algorithm

Figure 3 shows the experimental results of defect detection in different mechanical parts by using the proposed algorithm.

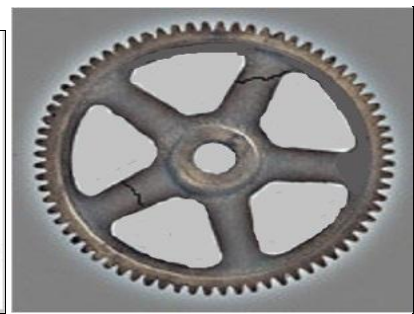

(g)

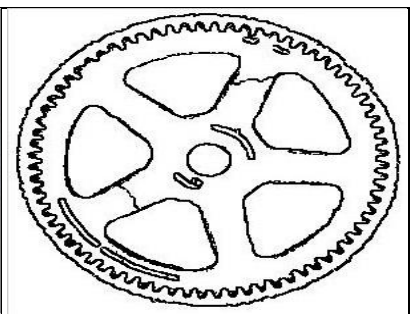

(h) image after applying Gaussian blur filter (c) image is obtained by the canny edge detection method (d) shows the image after applying the edge thresholding and noise filtering, it removes all noisy pixels and determines the actual edges of defect and (e) shows binary output image in which each and every defect

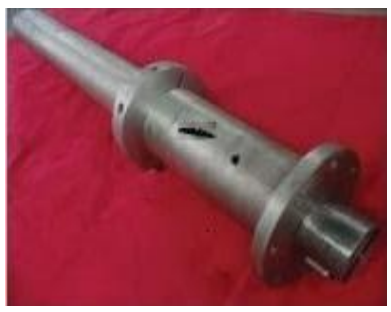

(a) Test image 1

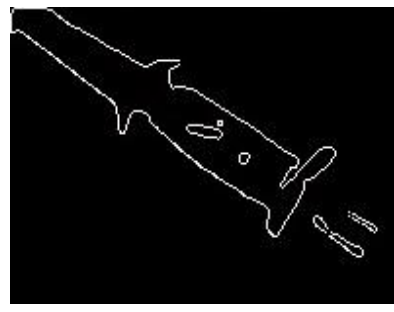

(c) Edge Detected Image

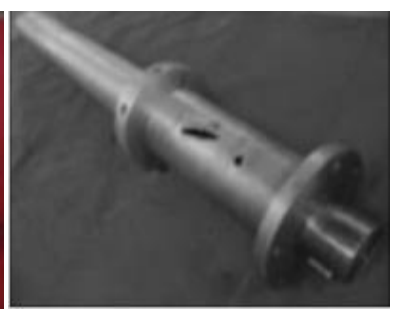

b) Preprocessed image

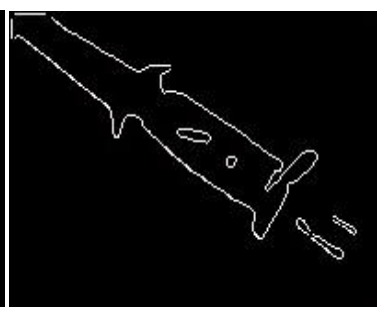

(d) Noise Filtered Image

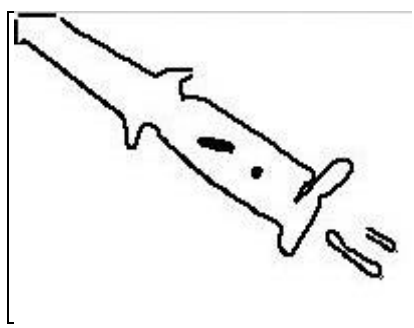

(e) Output Image

Fig 2: Result 1 of proposed algorithm steps

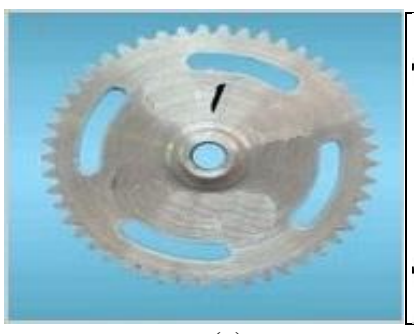

(c)

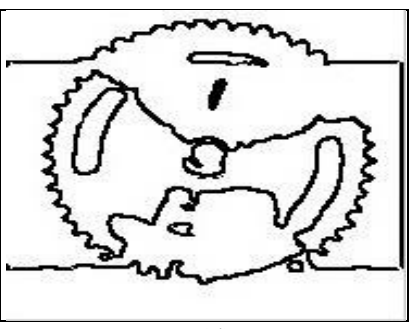

(d)
Figures (a), (c), (e), (g) represent the test images of different mechanical parts which shows the different defects of varied 
shape and size. The mechanical part images which we have tested in a proposed algorithm like gear, spare parts, screw, etc... Figures (b), (d), (f), (h) are the output images obtained after applying above mentioned algorithm steps.

Now, for the accurate detection of the defects, we have considered to measure the detection success rate for showing the improvement to visualizing the actual defect in images. Generally, detection success rate, also known as detection accuracy [14], is defined as i.e. Detection Success Rate= (Number of samples correctly detected /Total Number of samples).

Table 1. Total defect detection accuracy

\begin{tabular}{|c|c|l|l|c|}
\hline $\begin{array}{c}\text { Image } \\
\text { Type }\end{array}$ & $\begin{array}{c}\text { Total no } \\
\text { of } \\
\text { Samples }\end{array}$ & \multicolumn{3}{|c|}{ Detection Success Rate } \\
\hline $\begin{array}{c}\text { Mechanical } \\
\text { Part }\end{array}$ & 21 & $\begin{array}{l}\text { Sample } \\
\text { Identified }\end{array}$ & $\begin{array}{l}\text { Sample } \\
\text { Un- } \\
\text { identified }\end{array}$ & $\%$ \\
Surface & & 20 & 1 & 95.24 \\
\cline { 3 - 5 } Defects & & & & \\
\hline
\end{tabular}

The proposed method was tested on different mechanical part samples which contains single as well as multiple defects of varying shape. We have obtained more than $95 \%$ accuracy in a proposed algorithm (see Table 1). This accuracy depends on the number of samples taken. The results of the method suggest that this method is suitable for extracting various defects with accurate shape at the exact location in varied size images.

Table 2. Defect detection accuracy

\begin{tabular}{|l|c|c|}
\hline \multicolumn{1}{|c|}{ Author } & Technique & $\begin{array}{c}\text { Detection } \\
\text { accuracy }\end{array}$ \\
\hline $\begin{array}{l}\text { Hamid } \\
\text { Alimohamdi[6] }\end{array}$ & $\begin{array}{c}\text { Optimal Gabor } \\
\text { Filter method }\end{array}$ & $60-75 \%$ \\
\hline $\begin{array}{l}\text { Rashmi S } \\
\text { Deshmukh[7] }\end{array}$ & $\begin{array}{c}\text { Feature } \\
\text { extraction and }\end{array}$ & $90-95 \%$ \\
\hline K. N. & $\begin{array}{c}\text { Gabor Filter and } \\
\text { Gaussian Filter }\end{array}$ & $85-93 \%$ \\
\hline Proposed Work & Extraction & More than $95 \%$ \\
\hline
\end{tabular}

Table 2 shows the accuracy of defect detection of existing methods and proposed method in terms of percentage. In existing approaches, they are limited to predefined defect type and size .So, the proposed approach has the capability to solve this problem and also gives the high defect detection accuracy. Furthermore, it is efficient in detecting more than one defect as well as any type of defect with varying shape like crack, blow whole, spot etc. in images.

\section{CONCLUSIONS}

In this paper, based on the obtained edge information, a unique approach which utilizing statistics and shape properties are developed in order to fill the gap between two corresponding defect edges. That way, the defects becomes highlighted and extracted. The method can rapidly and effectively extract the each and every defect of varying shapes from the images which contain complex background and noise. We tested the performance of the proposed algorithm using different mechanical parts images which contain different defects. The experimental results show that the proposed approach gives the accurate detection of the defects with varied shape in an image. The proposed algorithm is able to find out the defects even if the images have changing background due to illumination. Thus, the algorithm has the capacity to be used to find defects in various types of images like fabric, metal, road etc. as well as accuracy of detection is maintained by using the proposed approach.

\section{REFERENCES}

[1] G. M. Atiqur Rahaman and Md. Mobarak Hossain, "Automatic Defect Detection and Classification technique from image: A Special Case using Ceramic Tiles", International Journal of Computer Science and Information Security, Vol. 1(1), May 2009.

[2] L. Tomczak , V. Mosorov, D. Sankowski, J. Nowakowski," Image Defect Detection Methods for Visual Inspection Systems", in IEEE International Conference on CAD Systems in Microelectronic, pp. 454-456, 2007.

[3] Ajay Kumar and Grantham Pang, "Defect Detection in textural materials using Gabor filters" ,IEEE Transactions on Industry Applications,2002, Vol. 38(2):425-440,2002.

[4] K. L. Mak, P. Peng, H.YK. Lau, "A real time computer vision systems for detecting defects in textile fabrics", IEEE International Conference on Industrial Technology, pp. $469-474,2005$.

[5] A. Serdaroglu, A. Ertuzun and A. Ercil, "Defect detection in textile fabric images using wavelet transforms and independent component analysis", Pattern Recognition and Image Analysis, 2006, Vol. 16(1):61-64.

[6] Hamid Alimohamdi and Alireza Ahmady, "Detecting skin defect of fruits using optimal Gabor wavelet filter", International conference on Digital image Processing by IEEE, pp.402-406, 2009.

[7] K. N. Sivabalan, Dr. D. Ghanadurai," Detection of defects in digital texture images using segmentation", International Journal of Engineering Science and Technology, 2010, vol.2 (10): 5187-5191.

[8] R. S. Deshmukh, Dr P R Deshmukh," Comparison Analysis For Efficient Defect Detection Algorithm For Gray Level Digital Images Using Median Filters Gabor Filter and ICA“, International Journal of Advanced Research in Computer Science and Software Engineering ,2012,vol.2(1).

[9] K. N. Sivabalan and DR. D. Gnanadurai, "Efficient Defect Detection Algorithm for gray level digital images using 
Gabor Wavelet Filter and Gaussian Filter", International Journal of Engineering Science and Technology, Vol. 3, No. 4,2011

[10] Gui-mei Zhang, Shao-ping Chen, Jia-ni Liao, "Otsu Image Segmentation Algorithm Based on Morphology and Wavelet Transformation", in IEEE International Conference on Computer Research and Development , vol. 1, pp. 279-283, 2011.

[11] Fari Muhammad Abubakar,"Study Of Image Segmentation By Using Edge Detection Techniques", International Journal of Engineering Research \& Technology, Vol. 1 Issue 9, November- 2012.
[12] Poonam deep Kaur and Raman Maini," Performance Evaluation of Various Thresholding Methods using Canny Edge Detector, International Journal of Computer Applications(IJCA),Vol.71 No.9,2013.

[13] Febriliyan Samopa and Akira Asano," Hybrid Image Thresholding Method using Edge Detection", IJCSNS International Journal of Computer Science and Network Security, VOL.9 No.4, 2009.

[14] H.Y.T. Ngan, G.K.H. Pang, N.H.C. Yung, "Automated fabric defect detection-a review", Image Vision Computing, Vol. 29 (7):442-458, 2011. 